\title{
Alveolar Fluid Neutrophil Elastase Activity in the Adult Respiratory Distress Syndrome Is Complexed to Alpha-2-macrog!obulin
}

\author{
Mark D. Wewers, Danuta J. Herzyk, and James E. Gadek \\ Pulmonary and Critical Care Division, Department of Medicine, Ohio State University, Columbus, Ohio 43210
}

\section{Abstract}

We characterized the elastase and antielastase activity of the alveolar fluid of seven patients with the adult respiratory distress syndrome (ARDS) and thirteen normal volunteers. Alpha-1-antitrypsin (A1AT) concentrations were 60-fold higher in ARDS as compared to normal lavage fluid $(2,140 \pm 498 \mathrm{nM} ; 36.1 \pm 4.2 \mathrm{nM}$, respectively). ARDS fluid antineutrophil elastase activity was also considerably higher than that of normals $(979 \pm 204 \mathrm{nM} ; 31.3 \pm 2.9 \mathrm{nM}$, respectively). Despite the antineutrophil elastase excess, 5 of 7 ARDS lavage samples contained elastase activity (mean, $6.1 \pm 2.4 \mathrm{pM}$ ) as assayed using low-molecular-mass substrate, while only 1 of 13 normal subjects had detectable elastase activity $(0.2 \mathrm{pM})(P$ $<0.01$, compared with ARDS). That this activity was due to alpha-2-macroglobulin (A2MG)-complexed neutrophil elastase was evidenced by (a) the Sephadex G-75 elution profile; (b) the inactivity against insoluble $\left[{ }^{3} \mathbf{H}\right]$ elastin; (c) the inhibitory profile with phenylmethylsulfonyl fluoride, methoxy-succinyl-alanyl-alanyl-prolyl-valyl-chloromethylketone, ethylene diamine tetraacetic acid, and A1AT; and (d) the immobilization by A2MG antibody bound to polystyrene plates. Furthermore, in agreement with the predicted affinity of A1AT and A2MG for neutrophil elastase, the ratio of A2MG to A1AT in the fluid (0.57\%) coincided with the ratio of the A2MG- to A1AT-complexed elastase (0.36\%). These findings suggest that the net lung protease-antiprotease balance in ARDS is shifted largely in favor of the antiproteases (chiefly A1AT), and that the antiproteases, A1AT and A2MG, have similar affinities for neutrophil elastase in vivo.

\section{Introduction}

The adult respiratory distress syndrome (ARDS) ${ }^{1}$ is a form of noncardiogenic pulmonary edema that occurs as a result of nonspecific damage to the alveolar capillary membrane (1-3). The damaged membrane's permeability is greatly increased

Address reprint requests to Dr. Mark D. Wewers, Pulmonary and Critical Care Division, Department of Internal Medicine, The Ohio State University, N325 Means Hall, 1654 Upham Drive, Columbus, OH 43210-1228. 1988.

Received for publication 14 March 1988 and in revised form 8 June

1. Abbreviations used in this paper: A1 AT, alpha-1-antitrypsin; A2MG, alpha-2-macroglobulin; ARDS, adult respiratory distress syndrome; CMK, methoxy-succinyl-ala-ala-pro-val chloromethyl ketone; MEOSAAPVNA, methoxysuccinyl-ala-ala-pro-val paranitroanalide.

J. Clin. Invest.

(c) The American Society for Clinical Investigation, Inc.

$0021-9738 / 88 / 10 / 1260 / 08 \$ \$ 2.00$

Volume 82, October 1988, 1260-1267 and the alveolar spaces are flooded with inflammatory cells and plasma proteins (4-8). Bronchoalveolar lavage studies in ARDS have consistently shown significant increases in neutrophils and neutrophil products such as elastase, myeloperoxidase, and collagenase (4, 6-8). Lavage studies have also shown large increases in epithelial lining fluid proteins (9). Not only are the total proteins elevated, but the fraction of high-molecular-mass proteins is also increased (9). It has been suggested that the loss of the alveolar capillary membrane's differential protein sieving is a specific marker for the damage that characterizes ARDS (9). For example, alpha-2-macroglobulin (A2MG), a $725 \mathrm{kD}$ serum protein which is almost undetectable in normal lavage fluid due to its large size, is a major constituent of ARDS lavage fluid. The smaller protein, alpha1-antitrypsin (A1AT) (52 kD), which is the major antiprotease of normal plasma and lung lining fluid (10), is also increased in ARDS lung lining fluid (11).

Given the potential for both protease excess (as evidenced by the large neutrophil influx) and for antiprotease excess (as evidenced by the large plasma protein influx), the net effect of the lung injury of ARDS on the protease-antiprotease balance in ARDS is uncertain. The studies that have addressed this question do not entirely agree. While the earliest studies found functional neutrophil elastase in the lavage fluid of a large percentage of ARDS patients $(4,6)$, three subsequent studies found no functional neutrophil elastase $(5,7,8)$. There are several possible explanations for the apparent discrepancy. The two studies demonstrating free elastase used samples from tracheal suctioning and small-volume lavages (i.e., $20 \mathrm{ml}$ total) $(4,6)$. In contrast, the three studies that show no free elastase used conventional lavage volumes (i.e., $100-300 \mathrm{ml}$ total) (5, $7,8)$. It is conceivable that the earlier studies sampled more of the central airways than the periphery of the lung and therefore represent elastase activity in a more central lung compartment. Other explanations include the possibilities that the patients with free elastase were sicker, later in their course, or had confounding problems such as oxygen toxicity or superinfection.

However, the increased influx of A2MG into the ARDS lung offers another possible solution. Although proteases trapped within A2MG are largely inactive against large-molecular-mass targets (e.g., A2MG-plasmin complexes have $<0.1 \%$ of the fibrinogen degrading activity of free plasmin) (12) A2MG-protease complexes may still retain function for low-molecular-mass substrates (13). Thus the disagreement in previous studies could potentially be reconciled by the fact that a significant portion of the neutrophil elastase released into the alveolar spaces in ARDS is complexed to A2MG. The introduction of relatively large amounts of A2MG, a high-affinity neutrophil elastase inhibitor normally excluded from the alveolar spaces, provides the potential for formation of A2MGneutrophil elastase complexes. If present, these complexes could provide functional elastase for assays that use low-molecular-mass elastase substrates, but not for assays that are 
based on the degradation of high-molecular-mass elastin degradation. Thus, these complexes could, in part, explain previous discrepant results relevant to protease-antiprotease balance in the lungs of ARDS patients.

In addition, the quantitation of A2MG-neutrophil elastase and A1AT-neutrophil elastase complexes provides the unique opportunity to compare the relative affinities of A1AT and A2MG for neutrophil elastase in an in vivo model of neutrophil elastase release. The measurement of the proportion of neutrophil elastase irreversibly complexed to A1AT and to A2MG in a locus of acute inflammation, allows the testing of in vitro kinetic studies in a human example of protease-antiprotease interactions.

Therefore, the purpose of the present investigation was to characterize the neutrophil elastase present in lavage fluid of ARDS patients for both function and relative binding to the lung inhibitors, A1AT and A2MG. These studies were compared with the findings in normal individuals. Studies were done with an emphasis upon evaluating lung lavage fluid in an unconcentrated state to avoid potential difficulties associated with the concentration and manipulation of lavage fluid. We report the detection of functional neutrophil elastase in lung lavage fluid of patients with ARDS as measured by a low-molecular-mass substrate. This elastase activity has no activity toward high-molecular-mass substrates such as A1AT or elastin. This functional elastase appears to be complexed to A2MG, which is a functional inhibitor in the lungs of patients with ARDS.

\section{Methods}

Study population. Seven patients with ARDS were identified at the Ohio State University Medical Intensive Care Unit. All patients fit the criteria of ARDS as previously described (8), i.e., (a) diffuse pulmonary infiltrates shown by chest roentgenography, $(b)$ a requirement for an $\mathrm{FIO}_{2}$ of $>0.5$, and $(c)$ a pulmonary capillary wedge pressure reading of $<18 \mathrm{mmHg}$.

Thirteen normal individuals (five nonsmokers and eight smokers) were evaluated as controls. Normal individuals were without recent respiratory infection, denied history of pulmonary disease and had normal physical examinations. Both patients and controls consented to bronchoalveolar lavage by written informed consent according to a protocol approved by the Ohio State University Human Subjects Review Committee. Table I outlines the characteristics of the study population and the bronchoalveolar lavage results.

Bronchoalveolar lavage. After topical anesthesia with $1 \%$ lidocaine, bronchoscopy was performed with a 4.8-mm-diameter Olympus BF-B2 flexible fiberoptic bronchoscope through the endotracheal tube of patients (transnasally in normal individuals). Bronchoalveolar lavage was performed using the standard $100-\mathrm{ml}$ lavage in five $20-\mathrm{ml}$ aliquots of sterile $0.9 \%$ saline from the lingular or right middle lobe segment as previously described (14). Lavage fluid was collected by gentle suctioning into a trap, and was pooled and stored on ice for processing within $1 \mathrm{~h}$ of the procedure.

Processing of the lavage fluid. Recovered bronchoalveolar lavage fluid was strained through several layers of surgical gauze to remove excess mucous and debris, and then centrifuged at $500 \mathrm{~g}$ for $15 \mathrm{~min}$. The supernatant was decanted and buffered with $1.0 \mathrm{M}$ Tris- $\mathrm{HCl}$ in sufficient quantity to provide a final concentration of $0.05 \mathrm{M} \mathrm{Tris-} \mathrm{HCl}$. Unconcentrated samples were frozen in $1-\mathrm{ml}$ aliquots at $-80^{\circ} \mathrm{C}$.

Table I. Comparison of Population and Lavage Fluid Returns

\begin{tabular}{|c|c|c|c|c|c|c|c|c|c|}
\hline \multirow[b]{2}{*}{ Subject } & \multirow[b]{2}{*}{ Sex } & \multirow[b]{2}{*}{ Age } & \multirow[b]{2}{*}{$\begin{array}{l}\text { Smoking } \\
\text { history }\end{array}$} & \multirow[b]{2}{*}{ BAL $^{\ddagger}$} & \multirow[b]{2}{*}{$\begin{array}{l}\text { Cells recovered } \\
\text { in } \mathrm{BAL}^{8}\end{array}$} & \multicolumn{3}{|c|}{ Cell types recovered } & \multirow[b]{2}{*}{ Elastas } \\
\hline & & & & & & $\begin{array}{c}\text { Alveolar } \\
\text { macrophages }\end{array}$ & Lymphocytes & Neutrophils & \\
\hline & & & packs/yr* & & $\times 10^{6}$ & & $\%$ & & $p M$ \\
\hline \multicolumn{10}{|l|}{ ARDS } \\
\hline J.B. & $\mathbf{F}$ & 43 & 60 & $100 / 33$ & 44.5 & 19 & 3 & 78 & 10.6 \\
\hline R.B. & $\mathbf{F}$ & 64 & NS & $100 / 44$ & 131.0 & 13 & 0 & 86 & 13.0 \\
\hline S.B. & $\mathrm{F}$ & 19 & NS & $100 / 40$ & 1.8 & 12 & 5 & 83 & 4.0 \\
\hline B.D. & $\mathbf{F}$ & 32 & NS & $100 / 55$ & 8.2 & 40 & 0 & 60 & 0.9 \\
\hline E.M. & $\mathbf{F}$ & 28 & 30 & $100 / 33$ & 4.3 & 35 & 0 & 65 & 0.0 \\
\hline F.R. & $\mathbf{M}$ & 47 & 60 & $100 / 57$ & 33.7 & 22 & 0 & 78 & 14.5 \\
\hline B.W. & $\mathbf{F}$ & 37 & NS & $100 / 49$ & 29.3 & 25 & 0 & 75 & 0.0 \\
\hline \multicolumn{10}{|c|}{ NORMAL } \\
\hline R.B. & $\mathbf{M}$ & 20 & NS & $100 / 65$ & 7.5 & 88 & 12 & 0 & 0 \\
\hline T.M. & $\mathbf{M}$ & 18 & NS & $100 / 65$ & 11.0 & 96 & 3 & 1 & 0 \\
\hline N.N. & $\mathbf{M}$ & 22 & NS & $200 / 85$ & 7.9 & 93 & 6 & 1 & 0 \\
\hline R.R. & $\mathrm{F}$ & 20 & NS & $100 / 63$ & 5.5 & 97 & 3 & 0 & 0 \\
\hline L.S. & $\mathbf{M}$ & 22 & NS & $200 / 125$ & 24.0 & 84 & 15 & 1 & 0 \\
\hline N.B. & $\mathbf{M}$ & 34 & 40 & $200 / 119$ & 88.9 & 93 & 2 & 5 & 0 \\
\hline K.C. & $F$ & 19 & 8 & $200 / 112$ & 36.8 & 97 & 1 & 2 & 0 \\
\hline W.E. & $\mathbf{M}$ & 38 & 40 & $200 / 100$ & 72.5 & 97 & 1 & 2 & 0 \\
\hline C.H. & $\mathrm{F}$ & 52 & 20 & $100 / 71$ & 21.2 & 92 & 8 & 0 & 0 \\
\hline P.K. & $F$ & 37 & 12 & $200 / 111$ & 150.0 & 90 & 0 & 10 & 0 \\
\hline L.M. & $\mathrm{F}$ & 30 & 10 & $200 / 79$ & 63.0 & 98 & 1 & 1 & 0 \\
\hline N.S. & $\mathbf{F}$ & 48 & 12 & $100 / 55$ & 59.3 & 86 & 4 & 10 & 0.15 \\
\hline D.T. & $\mathbf{M}$ & 50 & 30 & $180 / 87$ & 52.4 & 99 & 0 & 1 & 0 \\
\hline
\end{tabular}

* Packs of cigarettes smoked per day times the number of years smoked. ${ }^{\ddagger}$ BAL, Bronchoalveolar lavage fluid, volume (ml) saline instilled/volume $(\mathrm{ml})$ BAL fluid recovered. ${ }^{\S}$ Total number cells recovered in BAL fluid. 
The cell pellet was resuspended and washed twice in Hanks' balanced salt solution, and a small aliquot was used to prepare cytocentrifuge preparations using a Cytospin II (Shandon, UK). The slides were air dried and then stained with Diff-Quick (American Hospital Supply Corp., McGaw Park, IL). Cell differentials were performed by counting 200 cells.

Standardization of neutrophil elastase, alpha-1-antitrypsin, and alpha-2-macroglobulin. Purified human neutrophil elastase (Elastin Products Co., Pacific, MO) was dissolved into 0.1 M Hepes, pH 7.5, $0.5 \mathrm{M} \mathrm{NaCl}, 0.1 \%$ Brij detergent at approximately $125 \mu \mathrm{g} / \mathrm{ml}$ and stored in $0.1 \mathrm{ml}$ aliquots at $-80^{\circ} \mathrm{C}$. The activity of this material was determined indirectly as follows: it was titrated against a purified human A1AT standard (Sigma Chemical Co., St. Louis, MO) that had been functionally titrated against an active-site-titrated, highly purified preparation of porcine pancreatic elastase (ES438; Elastin Products Co.). The porcine pancreatic elastase was used for active-site titration because of its high degree of purity (single band on 12.5\% SDS-PAGE; data not shown). The azapeptide, $Z$-alanyl-alanyl-prolyl-Aza-alanyl-onitrophenol (Enzyme System Products, Livermore, CA) was used as modified by the procedure of Powers et al. (15). Briefly, $150 \mu \mathrm{l}$ of 0.5 $\mathrm{mM}$ azapeptide titrant in acetonitrile is added to $400 \mu \mathrm{l}$ of $0.1 \mathrm{M}$

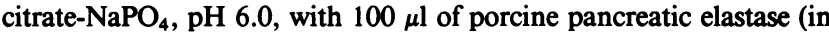
$0.2 \mathrm{M}$ tris- $\mathrm{HCl}, \mathrm{pH} 8.0,0.05 \mathrm{M} \mathrm{NaCl}$ ) or $100 \mu \mathrm{l}$ of the elastase buffer. The burst of nitrophenol is measured at $345 \mathrm{~nm}$, and the enzyme activity is determined by the extinction coefficient for nitrophenol (enzyme concentration $=$ burst $\mathrm{OD}_{345} / 6,250 \times$ dilution factor). The stock porcine pancreatic elastase activity was $45 \pm 4 \mu \mathrm{M}$, which was $85 \%$ of the antigenic material measured by the molar extinction coefficient of porcine pancreatic elastase $\left(E_{280}^{1 \%}=20.2\right)(16)$.

\section{Assay for functional elastase}

Low-molecular-mass substrate. To measure functional elastase at low concentrations such as might exist in unconcentrated bronchoalveolar lavage fluid, an assay specific for functional neutrophil elastase was developed to take advantage of the extreme long-term stability of the specific neutrophil elastase substrate, methoxysuccinyl-alanyl-alanylprolyl-valyl paranitroanalide (MEOSAAPVNA) (Sigma Chemical Co., St. Louis, MO) (17). Into individual wells of 96-well microtiter plates (Immulon 2; Dynatech Labs, Chantilly, VA), $0.190 \mathrm{ml}$ samples of neutrophil elastase or bronchoalveolar lavage were added to $0.05 \mathrm{ml}$ of $0.5 \mathrm{M}$ Hepes, pH 7.5, 0.5\% Brij detergent, and $0.01 \mathrm{ml}$ of $5 \mathrm{mM}$ MEOSAAPVNA. Baseline absorbance was read at $410 \mathrm{~nm}$ on a microplate reader (MR 600; Dynatech Labs) interfaced to an Apple IIe microcomputer and using Immunosoft software (Dynatech Labs) before the microtiter plates were covered with aluminum foil and incubated at $25^{\circ} \mathrm{C}$. The change in absorbance (final $\mathrm{A}_{410}-$ initial $\mathrm{A}_{410}$ ) was monitored at 24,48 , and $72 \mathrm{~h}$ for standardized amounts of neutrophil elastase, and the change in absorbance was found to be linear over this period for elastase concentrations $<50 \mathrm{pM}$.

High-molecular-mass substrate. The high-molecular-mass substrate assay for elastase was the solubilization of $\left[{ }^{3} \mathrm{H}\right]$ elastin.

Preparation of $\left[{ }^{3} \mathrm{H}\right]$ elastin. Tritium-labeled elastin was prepared as previously described (18). Briefly, $2.5 \mathrm{~g}$ of dry elastin powder (bovine ligamentum nuchae; Elastin Products Co.) was moistened with ethanol and suspended in $50 \mathrm{ml}$ of distilled water. The $\mathrm{pH}$ was adjusted to 9.2 with $100 \mathrm{mM} \mathrm{NaOH}$, and $\mathrm{NaB}_{3} \mathrm{H}_{4}(25 \mathrm{mCi})$ dissolved in $3 \mathrm{mM} \mathrm{NaOH}$, was added. After $10 \mathrm{~min}, 250 \mathrm{mg}$ of nonradioactive $\mathrm{NaBH}_{4}$ was added and mixed in a very-well-ventilated fume hood for $2 \mathrm{~h}$. The $\mathrm{pH}$ was then adjusted to 3.0 by the addition of acetic acid. Foaming was suppressed by a few drops of Antifoam-B emulsion (Sigma Chemical Co.). After 30 min of additional mixing, the elastin was collected by centrifugation at $10,000 \mathrm{~g}$ for $30 \mathrm{~min}$. The pellet was repeatedly washed with water until the supernatant radioactivity approached background values. The labeled elastin suspension $(50 \mathrm{mg} / \mathrm{ml}$; specific activity 3.6 $\times 10^{6} \mathrm{dpm} / \mathrm{mg}$ ) was aliquoted and stored at $-20^{\circ} \mathrm{C}$.

Elastin assay. The ability to solubilize elastin was measured by determining the amount of radioactivity solubilized after incubating $400 \mu \mathrm{l}$ of elastase standard or samples (prepared in $0.1 \mathrm{M}$ Hepes, $\mathrm{pH}$
7.5, 0.5 M NaCl, $0.1 \%$ Brij) with $100 \mu l$ of $\left[{ }^{3} \mathrm{H}\right]$ elastin $(5 \mathrm{mg} / \mathrm{ml})$ for $2 \mathrm{~d}$ $37^{\circ} \mathrm{C}$. After $5 \mathrm{~min}$ centrifugation in an Eppendorf Microfuge, the radioactivity of the supernatant was measured by dissolving $100 \mu \mathrm{l}$ of the supernatant in $4 \mathrm{ml}$ of Scinti Verse I (Fisher Scientific, Fair Lawn, $\mathrm{NJ}$ ) and counting in a Beckman liquid scintillation counter. This assay was able to detect elastase concentrations of $<5 \mathrm{pM}$.

Measurement of total protein. Total protein was measured in the unconcentrated lavage fluid by the Bio-Rad protein microassay procedure using a Dynatech MR 600 reader as referenced to an albumin standard.

Quantification of A1AT, A2MG, and neutrophil elastase. Enzymelinked immunosorbent assays (ELISA) were used. The A1AT ELISA is an indirect assay modified from the previously described assay (11). Briefly, purified A1AT (Sigma Chemical Co.) is coated onto 96-well Immunolon II plates (Dynatech Labs) at $200 \mathrm{ng} / \mathrm{ml}$ in carbonate buffer overnight. In separate 96-well plates standard A1AT and test samples are diluted in serial threefold dilutions in phosphate-buffered saline in $0.1 \%$ Tween and then incubated overnight with goat anti-human AlAT antibody (Sigma Chemical Co.). The antigen-coated dish is washed and then incubated with the sample-antibody mixture for $1 \mathrm{~h}$, washed, incubated with a 1:1,000 dilution of a peroxidase-conjugated, rabbit anti-goat immunoglobulin antibody, washed, and developed in o-phenylenediamine (Sigma Chemical Co.). The color is read at 490 nm on a MR 600 ELISA reader (Dynatech Labs) and analyzed by Immunosoft (Dynatech Labs) on an Apple Ile microcomputer using the Rodbard fit. This assay is sensitive to $100 \mathrm{ng} / \mathrm{ml}$ and is not affected by lavage fluid or serum. The standard A1AT was quantified by comparing it to a pooled sample of normal human serum ( $1.3 \mathrm{~g} /$ liter $)(19$, 20) on radial immunodiffusion plates (Behring Corp., La Jolla, CA).

The A2MG is measured similarly using purified A2MG (Sigma Chemical Co.) and a goat antihuman A2MG antibody (Sigma Chemical Co.) in an indirect ELISA.

The antigenic neutrophil elastase was measured by an indirect ELISA. Briefly, purified neutrophil elastase (Elastin Products Co.) was incubated overnight in 96-well Immulon 2 plates (Dynatech Labs) at $100 \mathrm{ng} / \mathrm{well}$, and the presence of elastase was detected by the ability of samples containing elastase to successfully inhibit the binding of a polyclonal rabbit anti-human neutrophil elastase antibody (Calbiochem-Behring, La Jolla, CA) to the elastase bound to the plate as described for A1AT.

Determination of anti-neutrophil elastase capacity of bronchoalveolar lavage fluid. The anti-neutrophil elastase capacity was measured by a modification of the technique of Meyer et al. (21). Briefly, increasing amounts of unconcentrated bronchoalveolar lavage fluid are incubated with $3 \mathrm{nM}$ of neutrophil elastase (Elastin Products Co.) in a volume of $1 \mathrm{ml}$ of $0.1 \mathrm{M}$ Hepes, $\mathrm{pH} 7.5,0.5 \mathrm{M} \mathrm{NaCl}, 0.1 \%$ Brij. Samples are incubated for $1 \mathrm{~h}$, which is more than five times the expected $t_{1 / 2}$ for the interaction (overnight incubations did not change the curves). Residual elastase activity was assayed by the addition of $0.1 \mathrm{mM}$ MEOSAAPVA (Sigma Chemical Co.) and recording the change of absorbance at $410 \mathrm{~nm}$ on a Beckman DU-50 spectrophotometer. For each sample six dilutions were done in triplicate. The inhibitory activity was determined by extrapolating the linear portion of the curve (using least squares fit) to the amount of lavage fluid needed to completely inhibit the active-site-titrated neutrophil elastase standard. This determination is accurate even in the face of A2MG found in lavage fluid. This inhibitory capacity technique was designed for use with serum in which A2MG represents as much as $10 \%$ of the neutrophil elastase inhibitory capacity (21). It is well suited for lavage fluid measurements since A2MG makes up $<1 \%$ of the molar elastase inhibitory equivalents of ARDS lavage fluid.

Characterization of elastase activity:inhibitor profile. The elastase activity (as measured by the low-molecular-mass substrate, MEOSAAPVNA) was characterized by its inhibition profile against $50 \mathrm{mM}$ EDTA, $5 \mathrm{mM}$ phenylmethylsulfonyl fluoride (PMSF), $0.2 \mathrm{mM}$ methoxy-succinyl-ala-ala-pro-val chloromethyl ketone (CMK), and $0.2 \mu \mathrm{M}$ A1AT. The active-site-titrated neutrophil elastase standard was similarly analyzed as a positive control. 
Chromatography of concentrated lavage fluid. To characterize the molecular size distribution of the elastase activity, selected lavage fluid samples were lyophilized, reconstituted to $1 \mathrm{ml}$, applied to a Sephadex G-75 column, and eluted in PBS at $4^{\circ} \mathrm{C}$. One-milliliter fractions were collected and saved for assay for elastase activity, and for ELISA antigenic assay for A1AT, A2MG, and neutrophil elastase as described above.

Statistical methods. All results are expressed as the mean and standard error of the mean $( \pm$ SEM). Comparisons between normals and ARDS patients are made using a paired Student's $t$ test.

\section{Results}

Measurement of elastase activity. The stability of the synthetic substrate, MEOSAAPVNA, allowed the extension of the elastase assay to $3 \mathrm{~d}$, in effect increasing the sensitivity of the assay. Fig. 1 demonstrates the linear nature and sensitivity of the low-molecular-mass substrate for elastase activity. As demonstrated, when the elastase was allowed to incubate with the substrate for $72 \mathrm{~h}$, there was a linear relationship with enzyme concentration up to $20 \mathrm{pM}$, and concentrations of elastase $<1$ pM could be detected.

Applying this assay to unconcentrated bronchoalveolar lavage fluid samples of 7 patients with ARDS and 13 normal subjects (see Table I for the general characteristics of the study population) there is a significant statistical difference between the ARDS patients and normal controls (Fig. 2). While only 1 of the 13 normal volunteers, a smoker, had detectable elastase activity $(0.2 \mathrm{pM})$, all but 2 of the ARDS patients had elastase activity, averaging $6.1 \pm 2.4 \mathrm{pM}, P<0.01$. Thus ARDS patients had at least a 30 -fold increase in elastase. There was a weak correlation between the percentage of neutrophils and the elastase activity, in the ARDS group $(r=0.66, P>0.1)$ and in the total study population $(r=0.71, P<0.001)$.

Measurement of alpha-1-antitrypsin. Using the A1AT ELISA, the concentrations of A1AT in the unconcentrated bronchoalveolar lavage fluid of patients with ARDS was 50-100 times higher than that of the normals (ARDS 2,140 $\pm 498 \mathrm{nM}$; normals $36.1 \pm 4.2 \mathrm{nM}$ ) (Fig. 3).

The anti-neutrophil elastase activity of the lavage fluid was also considerably higher in the ARDS group, compared with normals (Fig. 3). The ARDS group averaged 979 $\pm 204 \mathrm{nM}$ anti-neutrophil elastase activity (or $46 \%$ functional assuming activity totally due to A1AT). Conversely, the normal group

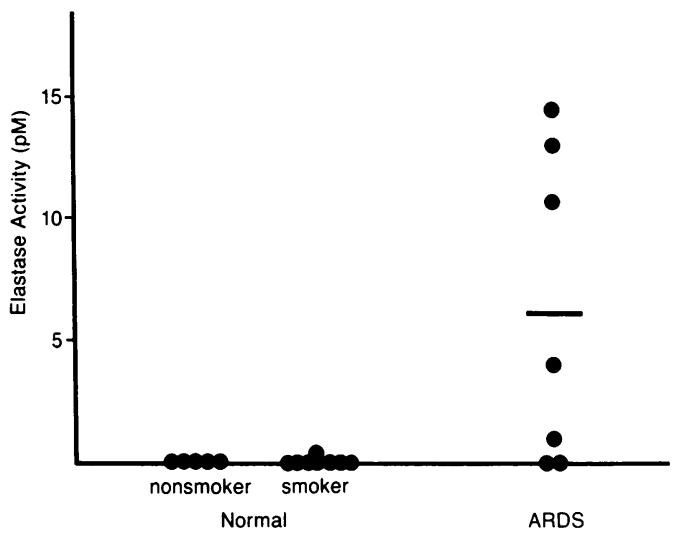

Figure 2. Comparison of neutrophil elastase activity in unconcentrated lavage fluid of ARDS patients and normal individuals. Aliquots of unconcentrated bronchoalveolar lavage fluid were assayed for neutrophil elastase activity using the low-molecular-mass substrate, MEOSAAPVNA, as described in Fig. 1. Each point represents the results of duplicate determinations. The bar represents the mean value for ARDS fluid of $6.1 \pm 2.2 \mathrm{pM}$.

averaged $31.3 \pm 2.9 \mathrm{nM}$ anti-neutrophil elastase activity (which corresponds to a functional A1AT activity of 83\%). Fig. 3 compares the differences between the antigenic and functional activities on a log scale. Thus, in spite of the fact that there is more than sufficient anti-neutrophil elastase activity in the ARDS group (i.e., about 30 times more than in normals, Fig. 3 ), there is also detectable elastase activity in this group (Fig. 2).

Sephadex G-75 chromatography of ARDS lavage fluid. In an effort to explain the apparent paradox, i.e., the presence of functional elastase activity in the face of elevated antielastase activity, two ARDS samples were concentrated by lyophilization and fractionated by G-75 sieve chromatography. Fig. 4 outlines the elution profile of proteins and elastase activity in a representative ARDS sample. Fig. $4 A$ demonstrates that the functional elastase activity coelutes with the initial high-molecular-mass protein peak of the column. This is in marked contrast to the antigenic elution of neutrophil elastase shown in Fig. $4 \mathrm{~B}$. Although the functional elastase coelutes with the antigenic $\mathrm{A} 2 \mathrm{MG}$, the antigenic elastase elutes entirely within the front edge of the AlAT peak. This is consistent with a

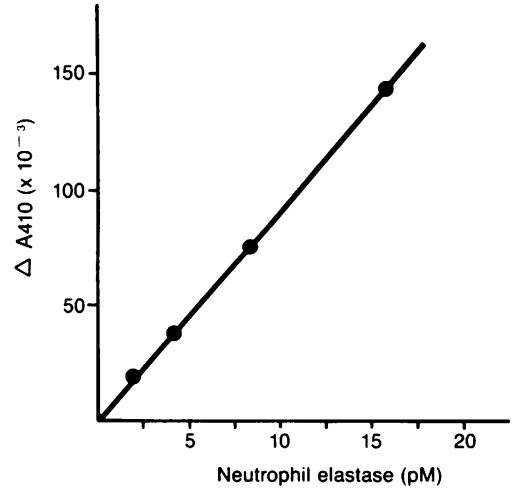

at $410 \mathrm{~nm}$ is determined from baseline to $72 \mathrm{~h}$ by daily recordings on microplate spectrophotometer.
Figure 1. Neutrophil elastase activity measurement using extended incubation with MEOSAAPVNA. Increasing amounts of active titrated neutrophil elastase are incubated in $0.2 \mathrm{mM}$ MEOSAAPVNA $(0.1 \mathrm{M}$ Hepes, pH 7.5, 0.1\% Brij) in 96-well flat-bottomed microtiter plates at room temperature. Change in absorbance

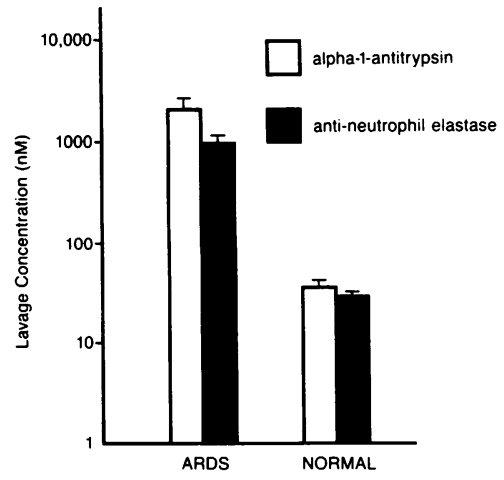

Figure 3. Comparison of A1AT and anti-neutrophil elastase activity in unconcentrated lavage fluid of ARDS patients and normal individuals. AIAT concentration was determined by ELISA as referenced to a purified A1AT standard (open bars). Anti-neutrophil elastase activity was determined by the ability of dilutions of unconcentrated lavage fluid to inhibit an active site titrated neutrophil elastase standard (closed bars) 


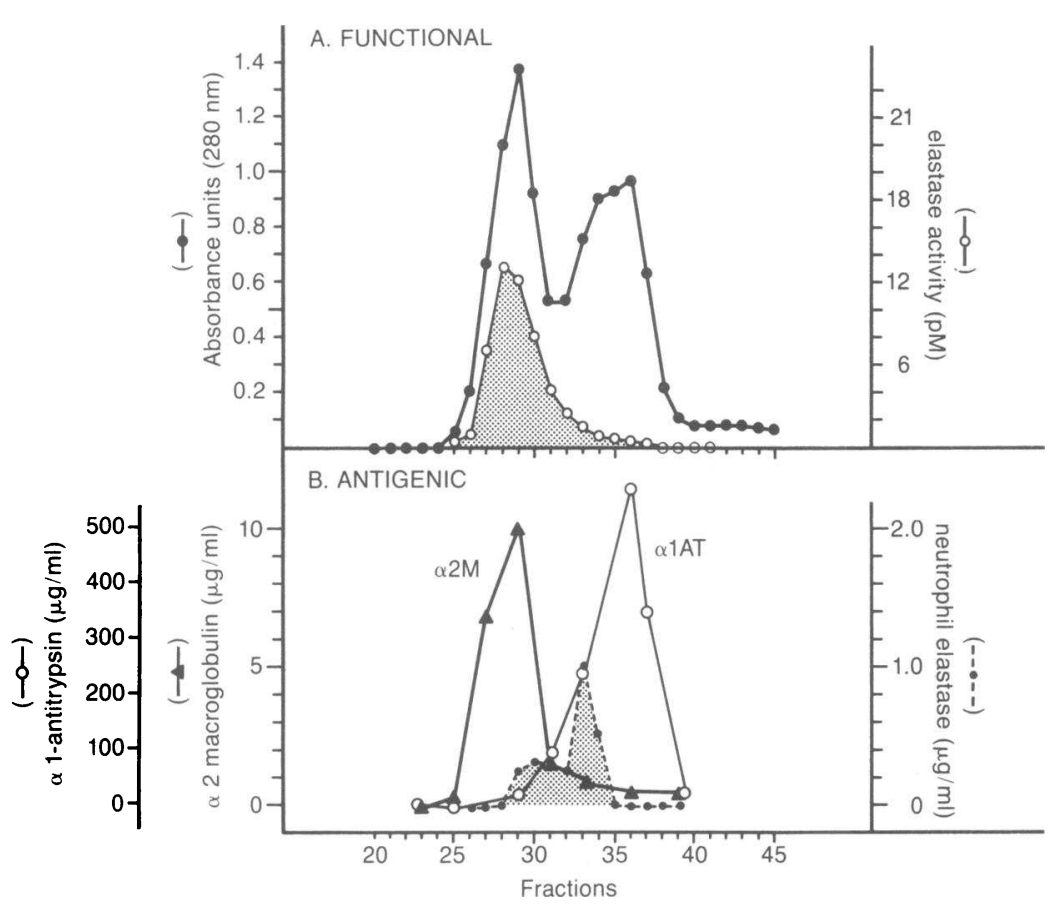

Figure 4. Sephadex G-75 elution profile of ARDS lavage fluid elastase. $A$. Elution of functional neutrophil elastase (open circles; shaded curve) measured by the extended MEOSAAPVNA assay compared to the protein elution as measured by the optical absorbance at $280 \mathrm{~nm}$ (closed circles). B. Elution of proteins measured antigenically by respective ELISA; A2MG (closed triangles); neutrophil elastase (closed circles; shaded curve); and A1AT (open circles). Fraction numbers are recorded on the abscissa. The scale for neutrophil elastase is expanded 250 -fold relative to the scale for A1AT; therefore the antigenic neutrophil elastase is completely encompassed by the antigenic AlAT peak. molecular mass less than A2MG $(725 \mathrm{kD})$ and greater than A1AT $(52 \mathrm{kD})$ as would be expected with A1AT-neutrophil elastase complexes. There was no antigenic or functional neutrophil elastase detectable at molecular masses less than $52 \mathrm{kD}$, confirming the absence of free neutrophil elastase. The elution profile for the other ARDS sample was identical in that the total elastase activity also eluted in the void volume.

ELISA data with standard neutrophil elastase analyzed alone and compared with neutrophil elastase complexed to A1AT or A2MG demonstrated that, while A1AT-complexed elastase was still accurately quantified by ELISA, that complexed to A2MG was not detectable (data not shown). This phenomenon has been previously described (22). In a parallel experiment, fractions eluted from the column were added to microtiter Immulon II plates that had been coated with antibody to A2MG. After washing, the presence of bound A2MGneutrophil elastase complexes were assessed by cleavage of the MEOSAAPVNA substrate in the microtiter wells. Only those fractions that contained functional elastase as analyzed fresh from the G-75 column demonstrated functional elastase activity that bound to the immobilized antibody to A2MG. Control incubations with uncomplexed neutrophil elastase standard did not demonstrate functional binding to the coated wells. Thus, taken together with the data that the functional elastase coeluted with A2MG, and that the antigenic neutrophil elastase coeluted with A1AT, these data suggest that the antigenic elastase represents A1AT-complexed neutrophil elastase while the functional elastase represents that elastase complexed to A2MG.

Summing the molar quantities of proteins eluted from the column confirms this comparison (Table II). Comparing the antigenic and functional elastase, the antigenic elastase peak represented 280 times the amount of functional elastase. This would suggest that 280 times more elastase is bound to AlAT than to A2MG. Interestingly, the molar ratio of A1AT to
A2MG in the eluate was 174:1 (i.e., in close agreement with the relative amount of elastase bound to A1AT as compared with A2MG). Expressed another way, the molar ratio of antigenic elastase to antigenic A1AT was $0.03 \%$, while the molar ratio for functional elastase to $\mathrm{A} 2 \mathrm{MG}$ was $0.02 \%$. This is consistent with the knowledge that A1AT and A2MG have similar affinities for neutrophil elastase $(12,23)$, and that neutrophil elastase will bind to either A1AT or A2MG based on the law of mass action.

Inhibitor profile. To further characterize the elastase activity, the active fractions from the Sephadex G-75 chromatography were incubated for $2 \mathrm{~h}$ with either EDTA (0.05 M), PMSF (0.005M), CMK (0.002 M), or A1 AT (0.001 mM) and assayed

Table II. Quantitative Relationships between Relevant Proteins in ARDS Lavage Fluid Eluted by Sieve Chromatography

\begin{tabular}{lc}
\hline \multicolumn{1}{c}{ Protein } & Amount \\
& pmol \\
A1AT & 82,000 \\
A2MG & 470 \\
NE (antigenic) & 28 \\
NE (functional) & 0.10 \\
\hline & Molar ratio* \\
& $\%$ \\
A2MG/A1AT & 0.57 \\
Funct./Antig.NE & 0.36 \\
Antig.NE/A1AT & 0.03 \\
Funct.NE/A2MG & 0.02 \\
\hline
\end{tabular}

Data generated from Fig. 4.

* Ratios of proteins expressed in molar quantities from data above. 
Table III. Inhibitor Profile of Elastase Activity

\begin{tabular}{lcr}
\hline & \multicolumn{2}{c}{ Percent inhibition } \\
\cline { 2 - 3 } \multicolumn{1}{c}{ Inhibitor } & $\begin{array}{c}\text { Neutrophil elastase } \\
\text { standard }\end{array}$ & Fraction* $^{*}$ \\
\hline None & 0 & 0 \\
EDTA $(50 m M)$ & 0 & 0 \\
PMSF $(5 m M)$ & 100 & 100 \\
CMK $(0.2 m M)$ & 100 & 100 \\
A1AT $(0.2 \mu M)$ & 100 & 1 \\
\hline
\end{tabular}

Elastase activity as measured with the extended MEOSAAPNVA assay (see Methods).

* Peak fraction of elastase activity from G-75 chromatography (10 pM).

for elastase by the low-molecular-mass substrate as previously described. A diluted sample of purified neutrophil elastase was used for comparison. Table III illustrates the results. While both PMSF and CMK were able to completely inhibit the ARDS lavage fluid elastase activity as well as the neutrophil elastase standard, A1AT was only able to inhibit the elastase standard and not the ARDS lavage fluid elastase activity. Neither sample was inhibited by EDTA. This data suggests that the activity is a serine protease, like neutrophil elastase, that is inaccessible to A1AT.

Functional profile. In addition to the inhibitor profile, the elastase activity was tested for its ability to degrade insoluble elastin. Fig. 5 outlines the sensitivity of this assay for purified neutrophil elastase and the results for the ARDS lavage fluid samples. While the assay is able to detect neutrophil elastase activity at $<5 \mathrm{pM}$ (the range of activity detected by the lowmolecular-mass substrate, Fig. 2, for the lavage fluid samples), lavage fluid elastinolytic activity is not detected. Thus the ARDS lavage fluid elastase is active against low-molecularmass substrate but not against macromolecular substrate (insoluble elastin).

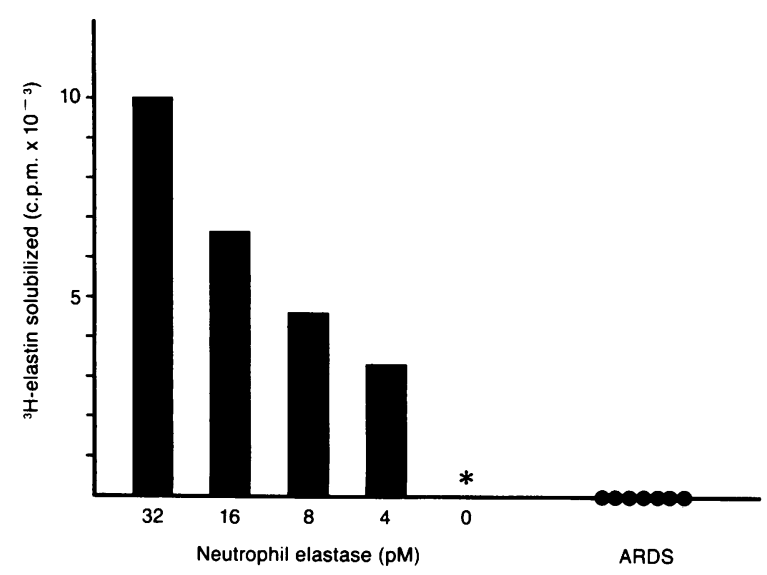

Figure 5. Ability of ARDS lavage fluid to degrade insoluble tritiated elastin. The bars represent the amount of $\left[{ }^{3} \mathrm{H}\right]$ elastin solubilized in response to a 48-h incubation with the neutrophil elastase standard. The asterisk denotes no solubilization for the control. The results for the seven unconcentrated ARDS lavage fluids are shown in the closed circles.

\section{Discussion}

Bronchoalveolar lavage studies of the alveolar milieu in ARDS characteristically demonstrate increases in both protein concentration and in inflammatory cell numbers in the lower respiratory tract. Protein levels in unconcentrated ARDS lavage fluid commonly range around $1 \mathrm{mg} / \mathrm{ml}(9)$, and neutrophil percentages are usually $60-90 \%(4-8)$ (normally $<0.15 \mathrm{mg} / \mathrm{ml}$ and $<5 \%$, respectively).

In agreement with the increase in neutrophils present in the lavage fluid, several studies have demonstrated increases in oxidative and proteolytic neutrophil mediators (4, 6-8). However, with respect to the presence of one particular protease, neutrophil elastase, there has been some controversy. Initial studies found functional neutrophil elastase to be present in concentrated lavage fluid $(4,6)$. This finding suggested the possible benefit from antiprotease therapy in those individuals with functional elastase within their lungs. However, subsequent studies have failed to demonstrate free elastase activity $(5,7,8)$. Since a clear understanding of the net protease/antiprotease balance is critical in the design of future therapeutic protocols for this syndrome, a critical evaluation of the function of the major inhibitors as compared to elastase measurements is warranted.

The present study takes advantage of newer, sensitive techniques and carefully titrated standards. These techniques permit the accurate measurement of elastase and antielastase activity in the unconcentrated lavage fluid from patients with ARDS, as compared with normals. This study addresses the controversy about neutrophil elastase activity in the lower respiratory tract of patients with ARDS, emphasizing the function of the major elastase inhibitors, A1AT and A2MG, as accessed by standard $100 \mathrm{ml}$ bronchoalveolar lavage.

This study confirmed the presence of markedly elevated elastase activity in the lungs of patients with ARDS when compared to both smoker and nonsmoker lung lining fluid. In the unconcentrated lavage fluid, five of seven ARDS samples contained elastase activity, while none of five nonsmoker and only one of eight smoker fluids sampled had any detectable elastase activity, using a neutrophil elastase-specific substrate. However, neutrophil elastase complexed to A2MG accounted for this activity. This was shown by several lines of investigation. First, when the active lavage fluid samples were concentrated and characterized by G-75 sieve chromatography, all the activity eluted at the void volume, consistent with a molecular mass of $>75 \mathrm{kD}$. Second, quantification of the A2MG present in the column fractions demonstrated that the elastase activity coeluted exactly with A2MG and distinctly separate from A1AT. Third, the elastase activity was not functional against a larger-molecular-mass substrate such as tritiated elastin, a specific characteristic of A2MG-complexed elastase $(12,13)$. Fourth, the activity was not inhibitable by A1AT or EDTA but was inhibited by PMSF, a serine protease inhibitor that has access to A2MG-trapped enzymes. Finally, this activity, which was demonstrated to be similar if not identical to neutrophil elastase (by its inhibitor profile and by its action on the neutrophil elastase specific substrate MEOSAAPVNA), was not recognized by neutrophil elastase antibody, yet was immobilized on polystyrene plates that were coated with antibodies to A2MG. 
In agreement with this characterization, the amounts of neutrophil elastase present in the antigenic form (presumably that complexed to A1AT) and in functional form (presumably that bound to A2MG) are entirely consistent with the molar ratio of these two elastase inhibitors in the lung lining fluid of ARDS patients. In the sample analyzed in detail in Fig. 4 and Table II, an analysis of the relative binding of neutrophil elastase to A1AT and A2MG provides indirect data about the relative function of these two inhibitors in vivo. The relative partitioning observed would have been predicted if one assumed a similar association rate constant (affinity) for the two inhibitors, and that the interactions follow the law of mass action. Therefore, previous in vitro studies that have demonstrated a similar affinity of A2MG and A1AT for neutrophil elastase $(12,23)$, have been substantiated in vivo in ARDS.

Thus, this study reconciles the apparent discrepancies that exist in the literature regarding the detection of neutrophil elastase activity in the lavage fluid of patients with ARDS. That is, since the studies that detected neutrophil elastase activity used a low-molecular-mass substrate, they may have detected A2MG-bound neutrophil elastase $(4,6)$, whereas the studies that failed to show activity used as the substrate insoluble elastin, which would not be accessible to elastase bound by A2MG $(5,7,8)$.

The A2MG-bound elastase concept, however, cannot entirely explain the previous discrepancy since one of the studies found evidence of functional neutrophil elastase by both lowmolecular-mass substrate and by elastinolysis of elastin-agar plates (6). It is probable that the relatively small volume of lavage $(20 \mathrm{ml})$ used in this study, or a difference in the stage of illness represents a partial explanation for the difference. Still however, $50 \%$ of the ARDS patients studied had the simultaneous detection of both functional A1AT and neutrophil elastase activity (6). This combination of both active AlAT and active elastase is difficult to explain by technical differences alone. Indeed this discrepancy provided the impetus for the hypothesis addressed in the present study, i.e., that the functional neutrophil elastase in ARDS lung lining fluid may be complexed to A2MG.

The significance of A2MG-neutrophil elastase complexes remains to be elucidated. The fact that these complexes have little effect on insoluble elastin suggests that they may not have a role in direct connective tissue injury. However, there is data to suggest that these complexes may function in immunomodulation. For example, A2MG-neutrophil elastase complexes do interact with specific receptors on alveolar macrophages (24), and there is preliminary data that this interaction may promote the release of neutrophil chemotactic factor(s) (25). Furthermore, there is evidence that in certain situations, A2MG-elastase complexes can produce injury. For example, Stone et al. suggest that porcine elastase-A2MG complexes instilled into hamsters may persist and promote lung injury (26). In addition, Galdston et al. have demonstrated that elastase-A2MG complexes may have activity on the elastin precursor, tropoelastin (27). Nevertheless, the elastin assay in the present study shows that the concentrations of elastase complexed to A2MG in the alveolar fluid of patients with ARDS are not able to degrade mature elastin. Thus A2MG-elastase complexes may have potential clinical consequences, but the inability of these complexes to degrade mature elastin suggests that elastin degradation is not a major consequence of their presence during the evolution of the acute lung injury of ARDS.

Although inactive against larger-molecular-mass substrates such as elastin, A2MG-enzyme complexes are known to be active against substrates smaller than serum trypsin inhibitor (28). In this context, the presence of the azurophilic granule product, neutrophil elastase, in ARDS lavage fluid suggests that other azurophilic granule proteases, such as cathepsin $G$ may also be present. A2MG has a high affinity for cathepsin $G$ and, therefore, it is likely that ARDS alveolar fluid also contains A2MG-cathepsin G complexes (13). It is known that cathepsin $\mathrm{G}$ is active against small peptides; for example, cathepsin $G$ can inactivate bradykinin (29) and can convert angiotensin I to angiotensin II $(30,31)$. Therefore, cathepsin G-A2MG complexes, if present, may play a role in the turnover of low-molecular-mass peptides in the lung in ARDS. Thus, although the significance of A2MG-enzyme complexes in the ARDS lung is not known, it is conceivable that such complexes may play roles independent of direct lung matrix injury.

In conclusion, ARDS is characterized by the influx of large quantities of protein into the alveolar space. Among the proteins present in increased amounts are A1AT, A2MG, and neutrophil elastase. Five of seven individuals with ARDS demonstrated neutrophil elastase activity in their bronchoalveolar lavage fluid in spite of the simultaneous detection of 30 times more antielastase function than is present in normal controls. This paradox was explained by the observation that while most of the neutrophil elastase is bound to A1AT, the functional elastase is primarily composed of that elastase complexed to A2MG and only detectable by low-molecular-mass substrate. Thus, in spite of the significantly increased burden of neutrophils and the detection of functional elastase activity, the protease/antiprotease balance in typical ARDS is shifted significantly in favor of the antiprotease, A1AT. At least in the ARDS individuals evaluated in this study, therapeutic modalities that propose to augment the antiprotease activity of the lower respiratory tract with A1AT appear to be unwarranted.

\section{References}

1. Ashbaugh, D. G., D. B. Bigelow, and T. L. Petty. 1967. Acute respiratory distress in adults. Lancet. ii:319-323.

2. Rinaldo, J. E., and R. M. Rogers. 1982. Adult respiratory distress syndrome: changing concepts of lung injury and repair. $N$. Engl. J. Med. 300:900-909.

3. Bone, R. C., P. F. Francis, and A. K. Pierce. 1976. Intravascular coagulation associated with the adult respiratory distress syndrome. Am. J. Med. 61:585-589.

4. Lee, C. T., A. M. Fein, M. Lippmann, H. Holtzman, P. Kimbel, and G. Weinbaum. 1981. Elastolytic activity in pulmonary lavage fluid from patients with adult respiratory-distress syndrome. N. Engl. J. Med. 304:192-196.

5. Fowler, A. A., S. Walchak, P. C. Giclas, P. H. Henson, and T. M. Hyers. 1982. Characterization of antiprotease activity in the adult respiratory distress syndrome. Chest. 81:50-51S.

6. McGuire, W. W., R. C. Spragg, A. B. Cohen, and C. G. Cochrane. 1982. Studies on the pathogenesis of the adult respiratory distress syndrome. J. Clin. Invest. 69:543-553.

7. Idell, S., U. Kucich, A. Fein, F. Kueppers, H. L. James, P. N. Walsh, G. Weinbaum, R. W. Colman, and A. B. Cohen. 1985. Neutrophil elastase-releasing factors in bronchoalveolar lavage from patients with adult respiratory distress syndrome. Am. Rev. Respir. Dis. 132:1098-1105. 
8. Weiland, J. E., W. B. Davis, J. F. Holter, J. R. Mohammed, P. M. Dorinsky, and J. E. Gadek. 1986. Lung neutrophils in the adult respiratory distress syndrome: clinical and pathophysiologic significance. Am. Rev. Respir. Dis. 133:218-225.

9. Holter, J. F., J. E. Weiland, E. R. Pacht, J. E. Gadek, and W. G. Davis. 1986. Protein permeability in the adult respiratory distress syndrome: loss of size selectivity of the alveolar epithelium. J. Clin. Invest. 78:1513-1522.

10. Wewers, M. D., M. A. Casolaro, and R. G. Crystal. 1987. Comparison of alpha-1-antitrypsin levels and antineutrophil elastase capacity of blood and lung in a patient with the alpha-1-antitrypsin phenotype null-null before and during alpha-1-antitrypsin augmentation therapy. Am. Rev. Respir. Dis. 135:539-543.

11. Gadek, J. E., G. A. Fells, R. L. Zimmerman, S. I. Rennard, and R. G. Crystal. 1981. Antielastases of the human alveolar structures: implications for the protease-antiprotease theory of emphysema. $J$. Clin. Invest. 68:889-898.

12. Harpel, P. C., and M. W. Mosseson. 1973. Degradation of human fibrinogen by plasma alpha-2-macroglobulin-enzyme complexes. J. Clin. Invest. 52:2175-2184.

13. Salvesen, G., G. D. Virca, and J. Travis. 1983. Interaction of alpha 2-macroglobulin with neutrophil and plasma proteinases. Ann. NY Acad. Sci. 421:316-326.

14. Hunninghake, G. W., J. E. Gadek, O. Kawanami, V. J. Ferrans, and R. G. Crystal. 1979. Inflammatory and immune processes in the human lung in health and disease: evaluation by bronchoalveolar lavage. Am. J. Pathol. 97:149-206.

15. Powers, J. C., R. Boone, D. L. Carroll, B. F. Gupton, C.-M. Kam, N. Nishino, M. Sakamoto, and P. M. Tuhy. 1984. Reaction of azapeptides with human leukocyte elastase and porcine pancreatic elastase: new inhibitors and active titrants. J. Biol. Chem. 259:42884294.

16. Shotton, D. M. 1969. Elastase. Methods Enzymol. 19:113-140.

17. Castillo, M. J., K. Nakajima, M. Zimmerman, and J. C. Powers. 1979. Sensitive substrates for human leukocyte and porcine pancreatic elastase: a study of the merits of various chromophoric and fluorogenic leaving groups in assays for serine proteases. Anal. Biochem. 99:53-64.

18. Banda, M. J., and Z. Werb. 1981. Mouse macrophage elastase: purification and characterization as a metalloproteinase. Biochem. J. 193:589-605.
19. Jeppson, J.-O., C.-B. Laurell, and M. Fagerhol. 1978. Properties of isolated human alpha-1-antitrypsin of Pi types M, S, and Z. Eur. J. Biochem. 83:143-153.

20. Pannell, R., D. Johnson, and J. Travis. 1974. Isolation and properties of human plasma alpha-1-proteinase inhibitor. Biochemistry 13:5439-5445.

21. Meyer, J.-F., J. Bieth, and P. Metais. 1975. On the inhibition of elastase by serum: some distinguishing properties of alpha-1-antitrypsin and alpha-2-macroglobulin. Clin. Chim. Acta. 62:43-53.

22. Arnon, R. 1967. Antigenic properties of proteolytic enzmes. In Antibodies to Biologically Active Molecules. B. Cinader, editor. Pergamon Press, Oxford, 153-179.

23. Beatty, K., J. Bieth, and J. Travis. 1980. Kinetics of association of serine proteinases with native and oxidized alpha-1-proteinase inhibitor and alpha-1-antichymotrypsin. J. Biol. Chem. 255:3931-3934.

24. Kaplan, J., and E. A. Keogh. 1983. Studies on the physiology of macrophage receptors for alpha-2-macroglobulin-protease complexes. Ann. NY Acad. Sci. 421:442-456.

25. Gadek, J. E., G. A. Fells, G. W. Hunninghake, R. L. Zimmerman, and R. G. Crystal. 1982. Regulation of alveolar macrophage function by active and inhibitor-bound neutrophil proteases. Am. Rev. Respir. Dis. 125:212A. (Abstr.)

26. Stone, P. J., J. D. Calore, G. L. Snider, and C. Franzblau. 1982. Role of alpha-macroglobulin-elastase complexes in the pathogenesis of elastase-induced emphysema in hamsters. J. Clin. Invest. 69:920-931.

27. Galdston, M., V. Levytska, I. E. Liener, and D. Y. Twumasi. 1979. Degradation of tropoelastin and elastin substrates by human neutropohil elastase, free and bound to alpha-2-macroglobulin in serum of the $\mathrm{M}$ and $\mathrm{Z}(\mathrm{Pi})$ phenotypes for alpha-1-antitrypsin. $\mathrm{Am}$. Rev. Respir. Dis. 119:435-441.

28. Travis, J., and G. S. Salvesen. 1983. Human plasma proteinase inhibitors. Annu. Rev. Biochem. 52:655-709.

29. Reilly, C. F., N. B. Schechter, and J. Travis. 1985. Inactivation of bradykinin and kallidin by cathepsin $\mathrm{G}$ and mast cell chymase. Biochem. Biophys. Res. Comm. 127:443-449.

30. Reilly, C. F., D. A. Tewksbury, N. M. Schechter, and J. Travis. 1982. Rapid conversion of angiotensin I to angiotensin II by neutrophil and mast cell proteinases. J. Biol. Chem. 257:8619-8622.

31. Wintroub, B. U., L. B. Klickstein, and K. W. K. Watt. 1981. A human neutrophil-dependent pathway for generation of angiotensin II: purification of the product and identification as angiotensin II. $J$. Clin. Invest. 68:484-490. 